\title{
Crosslinking of nucleotide binding domains improves the coupling efficiency of an $\mathrm{ABC}$ transporter
}

\author{
Chengcheng Fan ${ }^{1}$, Jens T. Kaiser ${ }^{1}$ and Douglas C. Rees ${ }^{1,2 *}$
}

${ }^{1}$ Division of Chemistry and Chemical Engineering, California Institute of Technology, Pasadena, CA 91125

${ }^{2}$ Howard Hughes Medical Institute, California Institute of Technology, Pasadena, CA 91125

${ }^{*}$ Corresponding author, email: dcrees@caltech.edu 


\section{Abstract}

ATP Binding Cassette (ABC) transporters often exhibit significant basal ATPase activity

3 in the absence of transported substrates. To investigate the factors that contribute to this

4 inefficient coupling of ATP hydrolysis to transport, we characterized the structures and functions

5 of variants of the bacterial Atm1 homolog from Novosphingobium aromaticivorans (NaAtm1),

6 including forms with disulfide crosslinks between the nucleotide binding domains. Unexpectedly,

7 disulfide crosslinked variants of NaAtm1 reconstituted into proteoliposomes not only transported

8 oxidized glutathione, but also exhibited more efficient coupling of ATP hydrolysis to GSSG

9 transport than the native transporter. These observations suggest that enhanced conformational

10 dynamics of reconstituted NaAtm1 may contribute to the inefficient use of ATP. Understanding

11 the origins of this uncoupled ATPase activity, and reducing the impact through disulfide

12 crosslinking or other protocols, will be critical for the detailed dissection of ABC transporter

13 mechanism to assure that the ATP dependent steps are indeed relevant to substrate

14 translocation. 


\section{Introduction}

Membrane transporters couple the translocation of ligands to a thermodynamically favorable driving force. A key feature of the transport mechanism is that these two processes ligand transport and the driving force - must be linked to minimize "short circuiting" of the transduction process (Tanford 1983, Hill 1989). In essence, transporters kinetically facilitate the coupled reaction while disfavoring the individual uncoupled reactions. One way to achieve coupling between the favored and unfavored reactions is when both processes proceed through a common set of conformational states so that they can only be accessed when the relevant components are both present (Rees and Howard 1999). Structure based mechanisms defining these coupling processes have been advanced for several transporters, including lac permease (Kaback 2015) and P-type ATPases (Palmgren and Nissen 2011).

In contrast to those systems, ATP Binding Cassette (ABC) transporters provide an interesting situation where the coupling mechanism is less well defined. The transport mechanism is generally described by the alternating access model involving inward-facing, occluded and outward-facing states, with the transitions between states coupled to the binding and hydrolysis of ATP, followed by product release (Hofmann et al. 2019). A striking feature of certain characterized $\mathrm{ABC}$ transporters, including exporters and importers (Lewinson and Livnat-Levanon 2017), is the significant uncoupled ATPase activity in the absence of substrate, reflected in high basal ATPase activities, and the inefficient coupling of ATP hydrolysis to substrate transport, reflected in the ratio of hydrolyzed ATP to translocated substrate (Table 1). While the general view of $A B C$ transporters is that the coupling ratio is $\sim 2$ ATP per transported substrate, in practice, ratios near this value have been reported for only a few transporters and more typically greatly exceed 2 . Understanding and overcoming the underlying causes of the 
generally poor coupling efficiencies is critical for advancing the quantitative mechanistic

40 understanding of $\mathrm{ABC}$ transporters.

To investigate the factors that contribute to the high basal ATPase activity and coupling inefficiencies of $A B C$ transporters, we characterized the structures and transport functions of variants of a bacterial homolog of the $A B C$ transporter of mitochondria (Atm1) from Novosphingobium aromaticavorans (NaAtm1). Atm1 is a homodimeric exporter that was first reported to be involved in iron-sulfur cluster biogenesis in yeast (Kispal et al. 1997). The human homologs of Atm1, ABCB6 and ABCB7 (Lill and Kispal 2001), have been implicated in iron homeostasis (Kelter et al. 2007, Cavadini et al. 2007, Pondarre et al. 2006, Kispal et al. 1997); the plant homolog, Atm3 from Arabidopsis, is important for both iron-sulfur cluster biogenesis (Bernard et al. 2009, Zuo et al. 2017) and molybdenum cofactor maturation (Teschner et al. 2010). While the physiological substrates for these transporters are not well defined, earlier studies (Kuhnke et al. 2006) suggested that glutathione (GSH, reduced; GSSG, oxidized forms) and derivatives, including persulfidated forms (Schaedler et al. 2014, Riedel et al. 2019), are the substrates for Atm1 homologues. Our initial structural and functional studies of the bacterial homolog support a role of Atm1 in in heavy metal detoxification, plausibly by export of metallated glutathione species (Lee et al. 2014).

In this report, we characterize the GSSG transport and ATPase activities of wildtype NaAtm1 and variants with disulfide crosslinked NBDs. Unexpectedly, a disulfide stabilized variant exhibited more efficient coupling between ATP hydrolysis and transport, while retaining a transport rate $60 \%$ of the wild type transporter. These findings suggest that the enhanced conformational dynamics of reconstituted NaAtm1 may contribute to the high basal ATPase rate and the inefficient coupling to substrate transport. 


\section{Results}

64

\section{Disulfide bond crosslinking}

The conformational state of an $\mathrm{ABC}$ transporter is reflected in the arrangement of the NBDs which can vary from well-separated in the inward-facing conformation to a fully dimerized state in the outward facing form. As an approach to addressing how the accessibility of different conformational states influences the transport cycle of NaAtm1, we introduced disulfide bridges at the dimerization interface between the NBDs to stabilize the transporter in conformational states with juxtaposed NBDs (Korkhov, Mireku, and Locher 2012). Through sequence and structural alignments of NaAtm1 to ABC transporters with dimerized NBD structures (Figure S1a), stabilized either by nucleotide binding (Dawson and Locher 2006) or a disulfide bridge (Korkhov, Mireku, and Locher 2012), we identified three residues, A527, S526 and T525, near the Walker-B motif where disulfide bonds could potentially form between the equivalent residues in the two NBDs following substitution with cysteine and oxidation. These three residues were separately mutated to cysteine in the natively cysteine-less homodimeric NaAtm1 to generate three single-site variants: NaA527C, NaS526C, and NaT525C. All variants exhibited similar crosslinking yields in the initial crosslinking tests (Figure S1b). The availability of these disulfide crosslinked variants provided an approach to address the functional and structural properties of NaAtm1 upon constraining the relative positions of the NBDs.

\section{Proteoliposome reconstitution}

To test the ATPase and transport functions of NaAtm1 in a membrane like environment, we separately reconstituted into proteoliposomes (PLS) wildtype NaAtm1, the cysteine variants (NaA527C, NaS526C, and NaT525C) and NaE523Q, the ATP-hydrolysis deficient variant with the $E$ to $Q$ mutation in the Walker B-motif (Moody et al. 2002) by following an established 
protocol (Geertsma et al. 2008). The incorporation efficiency of NaAtm1 into PLS was evaluated by running samples on SDS-PAGE of the total PLS and the supernatant following ultracentrifugation. With a final reconstitution efficiency above 95\% (Figure S2a), it was assumed full incorporation for subsequent analyses. Transporters reconstituted into PLS can adopt two possible orientations with the NBDs positioned either outside or inside of the liposomes. In these studies, we utilized transporters oriented with the NBDs on the outside to measure both the ATPase and the transport activities (Figure 1a). In contrast, only the ATPase activities could be measured for NaAtm1 purified in detergent (Figure 1b). Following collection of the PLSs, transport activities were measured with a glutathione reductase based enzymatic assay, and the ATPase activities were measured using a molybdate based colorimetric assay (Chifflet et al. 1988).

\section{Transport and ATPase activities of wildtype and NaAtm1 mutants}

We first established the transport assay with wildtype NaAtm1 reconstituted in PLS, with MgATP and GSSG present at physiological concentrations, $10 \mathrm{mM}$ and $2.5 \mathrm{mM}$, respectively (Bennett et al. 2009) on the outside of the PLS. Transport activity was quantitated by recovering the PLS and measuring the accumulation over time of GSSG inside the PLS (Figure S2b). The negative controls showed no measurable time dependent GSSG uptake, although it appeared that low levels of GSSG stick to PLS or liposomes. For wildtype NaAtm1, the uptake of GSSG

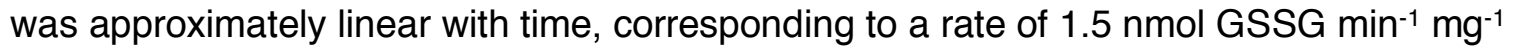
transporter (Figure 2a, S2b). Based on the NaAtm1 molecular weight of $133 \mathrm{kDa}$ (with $1 \mathrm{mg}=$ 7.5 nmole), neglecting orientation effects and assuming all the transporters are functionally active, this rate is equivalent to $\sim 0.2$ GSSG translocated per minute per transporter. Additionally, we measured the transport and ATPase activities of the different mutants for comparison to wildtype NaAtm1 (Figure 2). The wildtype NaAtm1 reconstituted in PLS 
showed an ATPase activity of $\sim 60 \mathrm{nmol} \mathrm{Pi} \mathrm{min-1} \mathrm{mg}^{-1}$ transporter with about 3-fold stimulation in the presence of $2.5 \mathrm{mM}$ GSSG to $\sim 180 \mathrm{nmol} \mathrm{Pi} \mathrm{min-1} \mathrm{mg}^{-1}$ transporter (Figure 2b); with NaAtm1 purified in detergent, the basal ATPase activity was $\sim 120 \mathrm{nmol} \mathrm{Pi} \mathrm{min-1} \mathrm{mg}^{-1}$ transporter and 2.5 mM GSSG only stimulated the ATPase activity by 2 -fold to $\sim 250 \mathrm{nmol} \mathrm{Pi} \mathrm{min}{ }^{-1} \mathrm{mg}^{-1}$ transporter (Figure 2c). NaA527C showed significantly reduced transport and ATPase activities in both PLS and detergent (Figure 2). Interestingly, NaS526C showed about $60 \%$ of wildtype transport

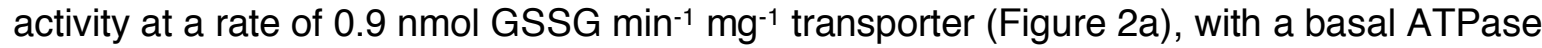
activity in PLS similar to wildtype with slight stimulation by GSSG (Figure 2b). NaT525C retained about $30 \%$ of wildtype transport activity with reduced ATPase activities in PLS and detergent (Figure 2). Lastly, NaE523Q exhibited little ATPase activities (Figure 2bc). Both NaA527C and NaE523Q exhibited a GSSG uptake rate about $\sim 10 \%$ of the wildtype protein transport activity despite their diminished ATPase activities (Figure 2a), which may reflect binding of substrate to the transporters.

\section{Inward-facing occluded conformations}

The structures of the different variants were determined by X-ray crystallography to assess the consequences of the disulfide crosslinks on the conformational state of NaAtm1. The crosslinked NaA527C form with bound MgADP crystallized in space group P1 with four transporters per asymmetric unit (Figure S3a). The initial crystallization trials were carried out with NaA527C containing ATP, but the same crystals were obtained with ADP in the crystallization conditions, suggesting these MgADP bound conformations could be achieved either by the slow hydrolysis of ATP or the direct binding of ADP. While each transporter adopted an inward-facing occluded conformation, two distinguishable states (\#1 and \#2) were evident. Three of the transporters were found in state \#1 (Figure 3a), while the fourth transporter in state \#2 exhibited a slightly more closed NBD dimer (Figure 3b). Given the moderate $3.7 \AA$ 
resolution and anisotropic diffraction, we confirmed the two distinct conformations from the locations of selenium sites in selenomethionine substituted protein crystals (Figure S3b). Clear electron density for the disulfide bridges was present in all four transporters (Figure S3c). The primary difference between these structures is reflected by a relative rotation of the NBD $\alpha$ helical subdomains about the molecular two-fold axis of the transporter (Figure S4a). The rootmean-square deviation (RMSD) between states \#1 and \#2 is $1.7 \AA$, while the RMSDs to the previous determined inward-facing structure of NaAtm1 are $2.1 \AA$ and $4.4 \AA$, respectively (Figure S4bcd).

Although GSSG was present in the crystallization conditions, ordered electron density for GSSG was not observed in the binding site. To assess whether this ligand was present, we co-crystallized NaA527C with a glutathione-mercury complex (GS-Hg) and collected data at the $\mathrm{Hg}$ absorption edge. Anomalous electron density peaks identifying $\mathrm{Hg}$ sites were found in all GSSG binding sites and were strongest in the two best resolved transporters in the asymmetric unit (Figure S4e). These sites were not observed in control studies using mercury compounds in the absence of glutathione, supporting the interpretation of GS-Hg complex presence in the binding site, which also reflects the binding capability of substrate at the binding site of NaA527C.

\section{Outward-facing occluded and occluded conformations}

NaS526C, NaT525C and NaE523Q were each crystallized by removing $\mathrm{MgCl}_{2}$ from the crystallization condition of NaA527C, followed by minor optimizations. The subsequent structure determinations revealed that these variants adopted similar ATP bound outward-facing occluded conformations (Figure 3c, S5ab). While there is clear electron density for the disulfide bond in the crystal structure of NaS526C (Figure S5c), the positions of the cysteine residues in NaT525C (separated by $13 \AA$ ) are incompatible with the presence of a disulfide bridge in the 
162 crystal structure of this variant (Figure S5d). Since no reductant was added, presumably the 163 uncrosslinked population of NaT525C was crystallized. The RMSDs between these different 164 outward-occluded structures are $\sim 0.5 \AA$ (Figure S5efg). As the periplasmic regions (residues $165 \quad 60-82$ and 284-300) were not well resolved, likely due to the lack of stabilizing crystal lattice 166 contacts, these loops were modeled based on previous structures and refined with low 167 occupancies. Despite the presence of GSSG or GS-Hg in the crystallization conditions, no 168 evidence for substrate binding was observed. In addition to these mutants, we were able to crystallize and determine the crystal 170 structure of a fully occluded state of wild type NaAtm1 with MgAMPPNP bound at $3.35 \AA$ 171 resolution (Figure $3 d$ ). This occluded structure shared similar overall architecture as the other 172 outward-facing occluded structures, with alignment RMSDs of $\sim 1 \AA$ (Figure S6). The main 173 distinction is in the periplasmic loop regions that were fully resolved in this electron density map. 
bioRxiv preprint first posted online Nov. 9, 2019; doi: http://dx.doi.org/10.1101/836676. The copyright holder for this preprint (which was not peer-reviewed) is the author/funder, who has granted bioRxiv a license to display the preprint in perpetuity.

It is made available under a CC-BY 4.0 International license.

175

176

177

178

179

180

181

182

183

184

185

186

187

188

189

190

191

192

193

194

195

196

197

198

\section{Discussion}

Within the framework of the alternating access mechanism, the transition between inward- and outward-facing conformations of $A B C$ transporters proceeds through various occluded states coupled to the binding and hydrolysis of ATP, followed by product release. A recent analysis of the TmrAB heterodimeric drug export established that the outward-facing conformation is stabilized by the binding of MgATP or the MgADP-Pi hydrolysis products, while Pi dissociation accompanies the return to the inward facing conformation (Hofmann et al. 2019). In this study, we have expanded the structurally characterized conformations of the $A B C$ exporter NaAtm1 from the initially determined inward-facing conformation to multiple occluded conformations through the use of disulfide-crosslinking of the NBDs and different nucleotides. As observed for previously characterized $A B C$ transporters, different conformations are associated with different ligands. For NaAtm1, we have observed that both the outward-facing occluded state and the fully occluded state are stabilized by binding of ATP, or the analog, MgAMPPNP, while the inward-facing and the inward-facing occluded conformations have been observed either nucleotide-free or with bound MgADP. The substrate GSSG has only been observed to bind to inward-facing conformations (Lee et al. 2014), at least at concentrations up to $5 \mathrm{mM}$.

Previous structural alignments of available exporters structures using a single subunit established that the major conformational differences between various states involve the movement of TM4 and TM5 toward the translocation pathway as the transporter adopts an outward-facing conformation (Lee et al. 2014). With structural alignment of all the NaAtm1 structures, changes in TM6 helices between the different conformations are also evident (Figure 4a). In the inward-facing and inward-facing occluded structures of NaAtm1, the TM6 helices are kinked, in contrast to the rather straight TM6 helices in the occluded structure and the more 
subtly bent TM6 helices in the outward-occluded and occluded structures of NaAtm1 and the outward-facing Sav1866 (Dawson and Locher 2006). The kinks in TM6 helices associated with the inward-facing conformation occur near two methionine residues, Met317 and Met320, that were previously noted to be involved in the binding of substrates in the inward-facing conformation (Lee et al. 2014). The bends in the TM6 helices observed in the outward-occluded conformations have shifted towards the $\mathrm{N}$-terminal residues by roughly a helical turn to residue Arg313, suggesting that the conformation of TM6 may be sensitive to the presence or absence of bound substrate by NaAtm1. These changes in TM6 differ from observations on ABCB1 where substrate binding is accompanied by kinks in the TM4 and TM10 helices (Alam et al. 2019, Alam et al. 2018).

The different conformational states of NaAtm1 are associated with changes in the substrate binding cavities (Figure 4b). The sizes of the cavities in the inward-facing occluded structures of $\mathrm{NaA527C}$ are similar to the original inward-facing structure, with sufficient space for substrate binding, as suggested by the GS-Hg anomalous maps (Figure S4e). In the occluded structure of NaAtm1 stabilized with MgAMPPNP, the periplasmic gate is fully closed. The three outward-facing occluded structures, represented by NaS526C, present slim access paths from the periplasmic end, but the pathways were not as wide open as in the Sav1866 outward-facing structure (Dawson and Locher 2006) (Figure 4b). Although the putative substrate binding cavities in the fully occluded and outward-occluded structures are of sufficient volume to accommodate GSSG (calculated as $1100-1500 \AA^{3}$ with CastP using a $3 \AA$ probe radius (Tian et al. 2018)), the addition of GSSG did not result in extra electron density in the substrate binding sites in the crystallographic studies. These observations suggest these structures may represent the post-translocation states of the transporter.

To assess the functional competence of the constructs generated for these studies, we measured the transport and ATPase activities for NaAtm1 and its variants. The activities were 
determined under physiological concentrations of MgATP and GSSG (10 mM and $2.5 \mathrm{mM}$,

225

226

227

228

229

230

231

232

233

234

235

236

237

respectively (Bennett et al. 2009)), and the observed values are within the range of values observed for other ABC transporters (Table 1). As a measure of coupling efficiency, we calculated the number of ATP hydrolyzed per translocated GSSG. This calculation may be performed in two ways (Table 2): (i) by taking the ratio of the total ATPase rate to the transport rate ("Total/transport" row in Table 2), or (ii) by taking the ratio of the stimulated ATPase rate (i.e. the additional ATPase rate in the presence of GSSG) to the transport rate (“Stimulated/transport" in Table 2). By focusing only on the increase in ATPase rate in the presence of transport substrate, the latter quantity presumably more accurately reflects the ATPase rate coupled to transport. Either way, it is apparent that the coupling of ATP hydrolysis to substrate transport, as defined by ATP hydrolyzed per substrate transported, is more efficient for the disulfide linked variants relative to the wild-type NaAtm1. Since disulfide bond formation in these variants was not quantitative, we cannot eliminate some contribution of uncrosslinked material to the observed ATPase and transport activities, but the improved coupling efficiency relative to wildtype NaAtm1 revealed that the crosslinked protein is indeed functional with distinct properties.

An unanticipated finding of this work is that introduction of a disulfide bond between NBDs can improve the coupling efficiency. The disulfide crosslink between NBDs might be expected to inhibit transport by restricting relevant conformational changes and preventing full opening of the NBDs, as seen in the structural comparisons of the crosslinked chimeric ABCB1 in an occluded conformation (Alam et al. 2018) to the uncrosslinked inward-facing conformation of ABCB1 (Aller et al. 2009). The observation that covalently linked NBDs can transport substrate more efficiently, albeit at a somewhat reduced rate, suggests that the poor coupling typically observed for $\mathrm{ABC}$ transporters may reflect that the reconstituted systems are too dynamic or "floppy". By restricting the conformational space through the introduction of disulfide 
crosslinks, the exploration of non-transport-relevant conformational states is apparently reduced, thereby improving the coupling efficiency. The functional relevance of the uncoupled ATPase activity can be viewed from two perspectives that are not necessarily mutually exclusive. On the one hand, the uncoupled ATPase activity could reflect some aspect of the proteoliposome reconstitution system used to study $A B C$ transporter function that does not faithfully mimic the native membrane, perhaps involving lipid composition. It is notable that significant variations in the coupling efficiency can be reported for a given transporter; for example, the coupling efficiency for the maltose transporter MalFGK in different studies ranges from 1.4 to over 3000 ATP/transported substrate (Table 1), which must arise from differences in the experimental protocols for reconstitution and transport characterization. On the other hand, the uncoupled activity could reflect the operation of two parallel, functionally relevant pathways for ATP hydrolysis that differ by the presence or absence of the transported substrate as proposed for certain ABC importers (Lewinson and Livnat-Levanon 2017) and ABC exporters (Hofmann et al. 2019). This behavior is in stark contrast to P-type transporters, a distinct class of ATP-dependent transporters where ATP hydrolysis is typically tightly coupled to transport (Palmgren and Nissen 2011). In this context, it may be relevant that P-type ATPases can exhibit turnover rates approaching $10^{4} \mathrm{~min}^{-1}$ (Skou 1998) which is considerably faster than the transport rates reported for ABC transporters (ranging between 1 to $1000 \mathrm{nmol} / \mathrm{min} / \mathrm{mg}$ (Table 1), equivalent to 0.1 to $100 \mathrm{~min}^{-1}$ for a $100 \mathrm{kDa}$ transporter). The lower turnover rates for $A B C$ transporters would reduce the metabolic impact of uncoupled ATPase activity relative to P-type ATPases, which for the $\mathrm{Na}^{+}, \mathrm{K}^{+}$ATPase represents a significant contribution to overall cellular energy consumption. The inefficient coupling of $A B C$ transporters is also reminiscent of the properties of binding protein independent variants of the maltose transporter that exhibit constitutive ATPase activity (Covitz et al. 1994); this suggests the uncoupled ATPase activity may be associated with the ability to transport 
bioRxiv preprint first posted online Nov. 9, 2019; doi: http://dx.doi.org/10.1101/836676. The copyright holder for this preprint (which was not peer-reviewed) is the author/funder, who has granted bioRxiv a license to display the preprint in perpetuity.

It is made available under a CC-BY 4.0 International license.

274 more weakly bound substrates. Understanding the origins of this uncoupling, and reducing the

275 impact through disulfide crosslinking or other protocols, will be critical for the detailed dissection

276 of the transport mechanism to assure that the ATP dependent steps are indeed relevant to

277 substrate translocation. 


\section{Author contributions}

279 C.F. and D.C.R designed the research; C.F. performed the research; C.F., J.T.K and D.C.R. 280 analyzed the data; and C.F and D.C.R prepared the manuscript.

281

282 Author identifications (ORCID)

283 Chengcheng Fan: 0000-0003-4213-5758

284 Jens T. Kaiser: 0000-0002-5948-5212

285 Douglas C. Rees: 0000-0003-4073-1185

286

287 Competing Interests

288 The authors declare no competing interests.

289

290 Data availability

291 Atomic coordinates were deposited in the Protein Data Bank with accession codes 6PAM

292 (NaA527C-MgADP), 6PAN (NaS526C-ATP), 6PAO (NaT525C-ATP), 6PAQ (NaE523Q-ATP)

293 and 6PAR (NaAtm1-MgAMPPNP). The raw data for ATPase and transport assays that support

294 the findings in Figure 2 and Table 2 are included in Table S6, and all the other data are 295 available from the corresponding author upon reasonable request. 


\section{Acknowledgments}

We thank the beamline staffs of the Stanford Synchrotron Radiation Lightsource beamline 12-2 and of the Advanced Photon Source GM/CA beamline for support during data collection. Discussions with Paul Adams, Gabriele Meloni, William Clemons, the organizers and speakers at the Cold Spring Harbor X-ray Method in Structural Biology Course (2018), the CCP4/APS School for Macromolecular Crystallography (2017) and the SBGrid/NE-CAT Phenix Workshop (2016) are gratefully acknowledged. We thank the Gordon and Betty Moore Foundation and the Beckman Institute for their generous support of the Molecular Observatory at Caltech. Use of the Stanford Synchrotron Radiation Lightsource, SLAC National Accelerator Laboratory, is supported by the U.S. Department of Energy, Office of Science, Office of Basic Energy Sciences under Contract No. DE-AC02-76SF00515. The SSRL Structural Molecular Biology Program is supported by the DOE Office of Biological and Environmental Research, and by the National Institutes of Health, National Institute of General Medical Sciences (including P41GM103393). GM/CA@APS has been funded in whole or in part with Federal funds from the National Cancer Institute (ACB-12002) and the National Institute of General Medical Sciences (AGM-12006). This research used resources of the Advanced Photon Source, a U.S. Department of Energy (DOE) Office of Science User Facility operated for the DOE Office of Science by Argonne National Laboratory under Contract No. DE-AC02-06CH11357. The Eiger 16M detector was funded by an NIH-Office of Research Infrastructure Programs, High-End Instrumentation Grant (1S10OD012289-01A1). 


\section{Materials and Methods}

\section{Mutagenesis and protein expression}

The gene encoding NaAtm1 (with GenBank accession code ABD27067) was previously cloned into a pJL-H6 ligation independent vector with 6-His tag on the carboxy-terminus (Lee et al. 2014, Lee and Kim 2009), and deposited in Addgene (catalog \#78308). All mutants were generated using Q5 Site-Directed Mutagenesis Kit (New England Biolabs). All constructs were overexpressed in Escherichia coli BL21-gold (DE3) cells (Agilent Technologies) using ZYM5052 autoinduction media (Studier 2005). Selenomethionine substituted proteins were overexpressed in Escherichia coli B834 (DE3) cells (Novagen) using PASM-5052 autoinduction media (Studier 2005). All cells were collected by centrifugation and stored at $-80{ }^{\circ} \mathrm{C}$ until use.

\section{Purification and crosslinking}

Frozen cell pellets of cysteine mutants were resuspended in lysis buffer containing 100 $\mathrm{mM} \mathrm{NaCl}, 20 \mathrm{mM}$ Tris, $\mathrm{pH}$ 7.5, 40 mM imidazole, $\mathrm{pH}$ 7.5, 5 mM $\beta$-mercaptoethanol (BME), 10 $\mathrm{mM} \mathrm{MgCl} 2,0.5 \%(\mathrm{w} / \mathrm{v}) \mathrm{n}$-dodecyl- $\beta$-D-maltopyranoside (DDM) (Anatrace), 0.5\% (w/v) octaethylene glycol monododecyl ether (C12E8) (Anatrace), lysozyme, DNase, and protease inhibitor tablet. The resuspended cells were lysed either by solubilizing by stirring for 3 hours at $4{ }^{\circ} \mathrm{C}$, or by using a M-110L pneumatic microfluidizer (Microfluidics). Unlysed cells and cell debris were removed by ultracentrifugation at $38,000 \mathrm{RPM}$ for 45 minutes at $4{ }^{\circ} \mathrm{C}$. The supernatant was collected and loaded onto a prewashed NiNTA column with NiNTA buffer A at $4{ }^{\circ} \mathrm{C}$. NiNTA buffer A contains $100 \mathrm{mM} \mathrm{NaCl}, 20 \mathrm{mM}$ Tris, $\mathrm{pH}$ 7.5, $50 \mathrm{mM}$ imidazole, $\mathrm{pH}$ 7.5, 5 mM BME, 0.05\% DDM and 0.05\% C12E8. Elution was achieved using the same buffer with $350 \mathrm{mM}$ imidazole. The eluted sample was then buffer exchanged to $100 \mathrm{mM} \mathrm{NaCl}, 20 \mathrm{mM}$ Tris, $\mathrm{pH}$ 7.5, 0.05\% DDM and $0.05 \%$ C12E8 (size exclusion chromatography (SEC) buffer). Oxidation of the 
introduced cysteines to form disulfide bonds was achieved by incubating buffer exchanged

342 protein with $1 \mathrm{mM} \mathrm{Cu}$ (II)-(1,10-phenanthroline) ${ }_{3}$ for 1 hour at $4{ }^{\circ} \mathrm{C}$. Crosslinked sample was then buffer exchanged into SEC buffer to remove the oxidant, and further purified by SEC on a using Amicon Ultra 15 concentrator (Millipore) with a molecular weight cutoff of $100 \mathrm{kDa}$ to 20$35 \mathrm{mg} / \mathrm{mL}$.

For cysteine-less constructs, the purified protein was prepared the same way as the cysteine constructs, but without BME. The eluted sample from NiNTA column was directly subjected to SEC without crosslinking or buffer exchange. Wildtype NaAtm1 was solubilized in lysis buffer containing 1\% DDM and purified in NiNTA and SEC buffers containing $0.1 \%$ DDM for crystallization in the occluded conformation with MgAMPPNP.

\section{Proteoliposome preparation and transport assay}

Proteoliposomes (PLS) were prepared by following published protocols for ABC

transporters (Geertsma et al. 2008), with an additional step of Biobeads addition to ensure detergent removal. The transport assay was conducted with NaAtm1 reconstituted in PLS in a 1 $\mathrm{mL}$ format at $37^{\circ} \mathrm{C}$. The reaction mixture contained PLS at $5 \mathrm{mg} / \mathrm{mL}, 10 \mathrm{mM} \mathrm{MgATP}, \mathrm{pH} 7.5$, different controls were also prepared in similar fashion. $150 \mu \mathrm{L}$ aliquots of the reaction mixture were taken every 15 minutes, added to $1 \mathrm{ml}$ cold transport buffer, and then ultracentrifuged at 70,000 RPM in a TLA 100.3 rotor in a Beckman Ultima benchtop ultracentrifuge for 10 minutes at $4{ }^{\circ} \mathrm{C}$. The pellets were washed 10 times with cold transport buffer, and then resuspended to $100 \mu$ with solubilization buffer ( $85 \mathrm{mM} \mathrm{NaCl}, 17 \mathrm{mM}$ Tris, $\mathrm{pH} 7.5$ and $2 \%$ sodium dodecanoyl sarcosine (Anatrace)). The samples were solubilized for 2 hours until the solution clarified 
before spinning down in the TLA 100 rotor to remove bubbles. $10 \mu \mathrm{L}$ samples were taken for GSSG quantification using the Glutathione Quantification Assay (Sigma-Aldrich). The rates were not corrected for orientation of NaAtm1 in PLS.

\section{ATPase assay}

The ATPase activity was determined by the molybdate based phosphate quantification method (Chifflet et al. 1988). Briefly, all reactions were performed in a $250 \mu \mathrm{L}$ scale with a final protein concentration of $0.05 \mathrm{mg} / \mathrm{ml}$ for both PLS and detergent at $37^{\circ} \mathrm{C} .50 \mu \mathrm{L}$ of reactions were taken every 5 minutes for 4 times, mixed with $50 \mu \mathrm{L}$ of $12 \%$ SDS in a 96-well plate at room temperature. $100 \mu \mathrm{L}$ of ascorbic acid/molybdate mix was added, incubated for 5 minutes before the addition of $150 \mu \mathrm{L}$ of citric acid/arsenite/acetic acid solution. The reaction was then incubated for 20 minutes at room temperature before reading at $850 \mathrm{~nm}$ with a Tecan plate reader. Reactions were done either in triplicates or sextuplicates, the absorbance measurements were plotted against time, and the final linear rates were fitted with nonlinear regression fit using Prism 8 . The rates were not corrected for orientation of NaAtm1 in PLS.

\section{Crystallizations and structural determinations}

NaA527C was crystallized in MemGold (Molecular Dimensions) condition \#68. Upon optimization of the crystallization conditions, including additive screens (Hampton Research), the best crystals were grown from $100 \mathrm{mM} \mathrm{NaCl}, 100 \mathrm{mM}$ Tris, $\mathrm{pH} 8.3,25 \mathrm{mM} \mathrm{MgCl}_{2}$, and $28 \%$ polyethylene glycol 2,000 monomethyl ether (PEG $2000 \mathrm{MME}$ ) with $20 \mathrm{mM}$ ATP at $20{ }^{\circ} \mathrm{C}$. The NaA527C crystallization sample was prepared at $20 \mathrm{mg} / \mathrm{mL}$ with $1 \mathrm{mM} \mathrm{ATP,} 5 \mathrm{mM}$ EDTA, with or without 5 mM GSSG. Crystals appeared in about 2 weeks and lasted for about 2 months. Crystals were harvested in cryoprotectant solutions containing $100 \mathrm{mM} \mathrm{NaCl}, 100 \mathrm{mM}$ Tris, pH 
8.3, $25 \mathrm{mM} \mathrm{MgCl}_{2}$, 28\% PEG $2000 \mathrm{MME}$ with PEG 400 at 10\%, 15\%, and $20 \%$ before flashfreezing in liquid nitrogen.

NaS526C, NaT525C and NaE523Q crystals were crystallized in the same condition as $\mathrm{NaA527C}$ except the removal of $\mathrm{MgCl}_{2}$ in the crystallization well solution. All crystallization samples were prepared with $1 \mathrm{mM}$ ATP and $5 \mathrm{mM}$ EDTA. The crystallization condition of NaT525C was further optimized with 200 mM of NDSB (non-detergents sulfobetaines)-221 using the Additive Screen (Hampton Research). The crystallization condition of NaE523Q was further optimized with $10 \mathrm{mM}$ dithiothreitol but without $20 \mathrm{mM}$ ATP in the crystallization conditions. Crystals of all three constructs were harvested in cryoprotectant solutions containing $100 \mathrm{mM} \mathrm{NaCl}, 100 \mathrm{mM}$ Tris, pH 8.3, 28\% PEG 2000 MME with PEG 400 at 10\%, 15\%, and $20 \%$ before flash-freezing in liquid nitrogen.

NaAtm1 purified in DDM was crystallized in MemChannel (Molecular Dimensions) condition \#29. The crystallization sample was prepared in the presence of $1 \mathrm{mM}$ AMPPNP, 2 $\mathrm{mM} \mathrm{MgCl}$, and presence and absence of $5 \mathrm{mM} \mathrm{GSSG}$. The condition was further optimized to $50 \mathrm{mM}$ ADA, pH 7.1, 8-10\% PEG 1000 and 8-10\% PEG 1500 at $20^{\circ} \mathrm{C}$ with protein at $8 \mathrm{mg} / \mathrm{ml}$. Crystals appears within a week. Crystals were harvested in cryoprotectant solutions containing 50 mM ADA, pH 7.1, 10\% PEG 1000 and 10\% PEG 1500 with PEG400 at 10\%, 15\%, and 20\% before flash-freezing in liquid nitrogen.

\section{Data collection and structure determination}

X-ray datasets were collected at the Stanford Synchrotron Radiation Laboratory beamline 12-2 using a Pilatus 6M detector with Blu-lce interface (McPhillips et al. 2002) and the Advanced Photon Source GM/CA beamline 23ID-B using an Eiger 16M detector with JBlulceEPICS interface (Stepanov et al. 2011). All datasets were processed and integrated with XDS (Kabsch 2010) and scaled with Aimless (Winn et al. 2011). 
For the NaA527C crystal structure, the first 3 transporters in the asymmetric unit were

415 identified by searching for multiple copies of the TMDs and NBDs using the original inward-

416 facing structure (PDB ID: 4MRN) with Phaser in Phenix (Adams et al. 2010). Due to the

417 relatively poor electron density for the fourth transporter, the helices of the TMDs were first built

418 using Find Helices and Strands in Phenix (Adams et al. 2010), then a full transporter from the

419 previously identified partial model was superposed onto the built helices in Coot (Emsley et al.

420 2010), which resulted in the misplacement of one NBD. The misplaced NBD was removed from

421 the model and it was then correctly placed using Molrep in CCP4 (Winn et al. 2011). For the

422 NaS526C structure, molecular replacement was carried out using Sav1866 (PDB ID: 2HYD)

423 with superposed NaAtm1 sequence as the input model for Phaser in Phenix (Adams et al.

424 2010). For the NaT525C, NaE523Q and NaAtm1 fully occluded structures, molecular

425 replacement was carried out using the NaS526C structure as the input model for Phaser in

426 Phenix (Adams et al. 2010). For all structures, experimental phase information from SeMet

427 datasets were obtained using with MR-SAD using AutoSol in Phenix (Adams et al. 2010).

428 Iterative refinement and model building cycles were carried out with phenix.refine in Phenix

429 (Adams et al. 2010), refmac in CCP4 (Winn et al. 2011) and Coot (Emsley et al. 2010), and the

430 final refinements were carried out with phenix.refine in Phenix (Adams et al. 2010). 


\section{References}

Adams, P. D., P. V. Afonine, G. Bunkoczi, V. B. Chen, I. W. Davis, N. Echols, J. J. Headd, L. W. Hung, G. J. Kapral, R. W. Grosse-Kunstleve, A. J. McCoy, N. W. Moriarty, R. Oeffner, R. J. Read, D. C. Richardson, J. S. Richardson, T. C. Terwilliger, and P. H. Zwart. 2010. "PHENIX: a comprehensive Python-based system for macromolecular structure solution." Acta Crystallographica Section D Biological Crystallography 66 (Pt 2):213-21. doi: 10.1107/S0907444909052925.

Alam, A., J. Kowal, E. Broude, I. Roninson, and K. P. Locher. 2019. "Structural insight into substrate and inhibitor discrimination by human P-glycoprotein." Science 363 (6428):753-756. doi: 10.1126/science.aav7102.

Alam, A., R. Kung, J. Kowal, R. A. McLeod, N. Tremp, E. V. Broude, I. B. Roninson, H. Stahlberg, and K. P. Locher. 2018. "Structure of a zosuquidar and UIC2-bound humanmouse chimeric ABCB1." Proceedings of the National Academy of Sciences of the United States of America 115 (9):E1973-E1982. doi: 10.1073/pnas.1717044115.

Aller, S. G., J. Yu, A. Ward, Y. Weng, S. Chittaboina, R. Zhuo, P. M. Harrell, Y. T. Trinh, Q. Zhang, I. L. Urbatsch, and G. Chang. 2009. "Structure of P-glycoprotein reveals a molecular basis for poly-specific drug binding." Science 323 (5922):1718-22. doi: 10.1126/science.1168750.

Bennett, B. D., E. H. Kimball, M. Gao, R. Osterhout, S. J. Van Dien, and J. D. Rabinowitz. 2009. "Absolute metabolite concentrations and implied enzyme active site occupancy in Escherichia coli." Nature Chemical Biology 5 (8):593-9. doi: 10.1038/nchembio.186.

Bernard, D. G., Y. Cheng, Y. Zhao, and J. Balk. 2009. "An allelic mutant series of ATM3 reveals its key role in the biogenesis of cytosolic iron-sulfur proteins in Arabidopsis." Plant Physiology 151 (2):590-602. doi: 10.1104/pp.109.143651.

Borths, E. L., B. Poolman, R. N. Hvorup, K. P. Locher, and D. C. Rees. 2005. "In vitro functional characterization of BtuCD-F, the Escherichia coli ABC transporter for vitamin B12 uptake." Biochemistry 44 (49):16301-9. doi: 10.1021/bi0513103.

Cavadini, P., G. Biasiotto, M. Poli, S. Levi, R. Verardi, I. Zanella, M. Derosas, R. Ingrassia, M. Corrado, and P. Arosio. 2007. "RNA silencing of the mitochondrial ABCB7 transporter in HeLa cells causes an iron-deficient phenotype with mitochondrial iron overload." Blood 109 (8):3552-9. doi: 10.1182/blood-2006-08-041632. 
Chavan, H., M. M. Khan, G. Tegos, and P. Krishnamurthy. 2013. "Efficient purification and reconstitution of ATP binding cassette transporter B6 (ABCB6) for functional and structural studies." Journal of Biological Chemistry 288 (31):22658-69. doi: 10.1074/jbc.M113.485284.

Chen, J., S. Sharma, F. A. Quiocho, and A. L. Davidson. 2001. "Trapping the transition state of an ATP-binding cassette transporter: evidence for a concerted mechanism of maltose transport." Proceedings of the National Academy of Sciences of the United States of America 98 (4):1525-30. doi: 10.1073/pnas.041542498.

Chifflet, S., A. Torriglia, R. Chiesa, and S. Tolosa. 1988. "A method for the determination of inorganic phosphate in the presence of labile organic phosphate and high concentrations of protein: application to lens ATPases." Analytical Biochemistry 168 (1):1-4.

Covitz, K. M., C. H. Panagiotidis, L. I. Hor, M. Reyes, N. A. Treptow, and H. A. Shuman. 1994. "Mutations that alter the transmembrane signalling pathway in an ATP binding cassette $(A B C)$ transporter." EMBO Journal 13 (7):1752-9.

Davidson, A. L., and H. Nikaido. 1990. "Overproduction, solubilization, and reconstitution of the maltose transport system from Escherichia coli." Journal of Biological Chemistry 265 (8):4254-60.

Dawson, R. J., and K. P. Locher. 2006. "Structure of a bacterial multidrug ABC transporter." Nature 443 (7108):180-5. doi: 10.1038/nature05155.

Dean, D. A., A. L. Davidson, and H. Nikaido. 1989. "Maltose transport in membrane vesicles of Escherichia coli is linked to ATP hydrolysis." Proceedings of the National Academy of Sciences of the United States of America 86 (23):9134-8. doi: 10.1073/pnas.86.23.9134.

Dong, M., F. Penin, and L. G. Baggetto. 1996. "Efficient purification and reconstitution of Pglycoprotein for functional and structural studies." Journal of Biological Chemistry 271 (46):28875-83. doi: 10.1074/jbc.271.46.28875.

Emsley, P., B. Lohkamp, W. G. Scott, and K. Cowtan. 2010. "Features and development of Coot." Acta Crystallographica Section D Biological Crystallography 66 (Pt 4):486-501. doi: 10.1107/S0907444910007493.

Eytan, G. D., R. Regev, and Y. G. Assaraf. 1996. "Functional reconstitution of P-glycoprotein reveals an apparent near stoichiometric drug transport to ATP hydrolysis." Journal of Biological Chemistry 271 (6):3172-8. doi: 10.1074/jbc.271.6.3172. 
Geertsma, E. R., N. A. Nik Mahmood, G. K. Schuurman-Wolters, and B. Poolman. 2008. "Membrane reconstitution of $\mathrm{ABC}$ transporters and assays of translocator function." Nature Protocol 3 (2):256-66. doi: 10.1038/nprot.2007.519.

Hill, Terrell L. 1989. Free Energy Transduction and Ciochemical Cycle Kinetics: Springer, New York, NY.

Hofmann, S., D. Januliene, A. R. Mehdipour, C. Thomas, E. Stefan, S. Bruchert, B. T. Kuhn, E. R. Geertsma, G. Hummer, R. Tampe, and A. Moeller. 2019. "Conformation space of a heterodimeric ABC exporter under turnover conditions." Nature 571 (7766):580-583. doi: 10.1038/s41586-019-1391-0.

Kaback, H. R. 2015. "A chemiosmotic mechanism of symport." Proceedings of the National Academy of Sciences of the United States of America 112 (5):1259-64. doi: 10.1073/pnas.1419325112.

Kabsch, W. 2010. "Xds." Acta Crystallographica Section D Biological Crystallography 66 (Pt 2):125-32. doi: 10.1107/S0907444909047337.

Kelter, G., D. Steinbach, V. B. Konkimalla, T. Tahara, S. Taketani, H. H. Fiebig, and T. Efferth. 2007. "Role of transferrin receptor and the $A B C$ transporters $A B C B 6$ and $A B C B 7$ for resistance and differentiation of tumor cells towards artesunate." PLoS One 2 (8):e798. doi: 10.1371/journal.pone.0000798.

Kispal, G., P. Csere, B. Guiard, and R. Lill. 1997. "The ABC transporter Atm1p is required for mitochondrial iron homeostasis." FEBS Letters 418 (3):346-50.

Korkhov, V. M., S. A. Mireku, and K. P. Locher. 2012. "Structure of AMP-PNP-bound vitamin B12 transporter BtuCD-F." Nature 490 (7420):367-72. doi: 10.1038/nature11442.

Kuhnke, G., K. Neumann, U. Muhlenhoff, and R. Lill. 2006. "Stimulation of the ATPase activity of the yeast mitochondrial ABC transporter Atm1p by thiol compounds." Molecular Membrane Biology 23 (2):173-184.

Lee, J., and S. H. Kim. 2009. "High-throughput T7 LIC vector for introducing C-terminal polyhistidine tags with variable lengths without extra sequences." Protein Expression and Purification 63 (1):58-61. doi: 10.1016/j.pep.2008.09.005.

Lee, J. Y., J. G. Yang, D. Zhitnitsky, O. Lewinson, and D. C. Rees. 2014. "Structural basis for heavy metal detoxification by an Atm1-type ABC exporter." Science 343 (6175):1133-6. doi: 10.1126/science.1246489. 
Lewinson, O., and N. Livnat-Levanon. 2017. "Mechanism of Action of ABC Importers: Conservation, Divergence, and Physiological Adaptations." Journal of Molecular Biology 429 (5):606-619. doi: 10.1016/j.jmb.2017.01.010.

Lill, R., and G. Kispal. 2001. "Mitochondrial ABC transporters." Research in Microbiology 152 (34):331-40.

Lycklama, A. Nijeholt J. A., R. Vietrov, G. K. Schuurman-Wolters, and B. Poolman. 2018. "Energy Coupling Efficiency in the Type I ABC Transporter GInPQ." Journal of Molecular Biology 430 (6):853-866. doi: 10.1016/j.jmb.2018.02.001.

Manolaridis, I., S. M. Jackson, N. M. I. Taylor, J. Kowal, H. Stahlberg, and K. P. Locher. 2018. "Cryo-EM structures of a human ABCG2 mutant trapped in ATP-bound and substratebound states." Nature 563 (7731):426-430. doi: 10.1038/s41586-018-0680-3.

McPhillips, T. M., S. E. McPhillips, H. J. Chiu, A. E. Cohen, A. M. Deacon, P. J. Ellis, E. Garman, A. Gonzalez, N. K. Sauter, R. P. Phizackerley, S. M. Soltis, and P. Kuhn. 2002. "Blu-Ice and the Distributed Control System: software for data acquisition and instrument control at macromolecular crystallography beamlines." Journal of Synchrotron Radiation 9 (Pt 6):401-6. doi: 10.1107/s0909049502015170.

Moody, J. E., L. Millen, D. Binns, J. F. Hunt, and P. J. Thomas. 2002. "Cooperative, ATPdependent association of the nucleotide binding cassettes during the catalytic cycle of ATP-binding cassette transporters." Journal of Biological Chemistry 277 (24):21111-4. doi: 10.1074/jbc.C200228200.

Nikaido, K., and G. F. Ames. 1999. "One intact ATP-binding subunit is sufficient to support ATP hydrolysis and translocation in an $\mathrm{ABC}$ transporter, the histidine permease." Journal of Biological Chemistry 274 (38):26727-35. doi: 10.1074/jbc.274.38.26727.

Palmgren, M. G., and P. Nissen. 2011. "P-type ATPases." Annual Review of Biophysics 40:24366. doi: 10.1146/annurev.biophys.093008.131331.

Patzlaff, J. S., T. van der Heide, and B. Poolman. 2003. "The ATP/substrate stoichiometry of the ATP-binding cassette (ABC) transporter OpuA." Journal of Biological Chemistry 278 (32):29546-51. doi: 10.1074/jbc.M304796200.

Pondarre, C., B. B. Antiochos, D. R. Campagna, S. L. Clarke, E. L. Greer, K. M. Deck, A. McDonald, A. P. Han, A. Medlock, J. L. Kutok, S. A. Anderson, R. S. Eisenstein, and M. D. Fleming. 2006. "The mitochondrial ATP-binding cassette transporter Abcb7 is essential in mice and participates in cytosolic iron-sulfur cluster biogenesis." Human Molecular Genetics 15 (6):953-64. doi: 10.1093/hmg/ddl012. 
Rees, D. C., and J. B. Howard. 1999. "Structural bioenergetics and energy transduction mechanisms." Journal of Molecular Biology 293 (2):343-50. doi: 10.1006/jmbi.1999.3005.

Riedel, S., B. Siemiatkowska, M. Watanabe, C. S. Muller, V. Schunemann, R. Hoefgen, and S. Leimkuhler. 2019. "The ABCB7-Like Transporter PexA in Rhodobacter capsulatus Is Involved in the Translocation of Reactive Sulfur Species." Frontiers in Microbiology 10:406. doi: 10.3389/fmicb.2019.00406.

Schaedler, T. A., J. D. Thornton, I. Kruse, M. Schwarzlander, A. J. Meyer, H. W. van Veen, and J. Balk. 2014. "A conserved mitochondrial ATP-binding cassette transporter exports glutathione polysulfide for cytosolic metal cofactor assembly." Journal of Biological Chemistry 289 (34):23264-74. doi: 10.1074/jbc.M114.553438.

Skou, J. C. 1998. "The Identification of the Sodium-Potassium Pump (Nobel Lecture)." Angewandte Chemie International Edition in English 37 (17):2320-2328. doi: 10.1002/(SICI)1521-3773(19980918)37:17<2320::AID-ANIE2320>3.0.CO;2-2.

Stepanov, S., O. Makarov, M. Hilgart, S. B. Pothineni, A. Urakhchin, S. Devarapalli, D. Yoder, M. Becker, C. Ogata, R. Sanishvili, N. Venugopalan, J. L. Smith, and R. F. Fischetti. 2011. "JBlulce-EPICS control system for macromolecular crystallography." Acta Crystallographica Section D Biological Crystallography 67 (Pt 3):176-88. doi: 10.1107/S0907444910053916.

Studier, F. William. 2005. "Protein production by auto-induction in high-density shaking cultures." Protein Expression and Purification 41 (1):207-234. doi: 10.1016/j.pep.2005.01.016.

Tanford, C. 1983. "Mechanism of free energy coupling in active transport." Annual Review of Biochemistry 52:379-409. doi: 10.1146/annurev.bi.52.070183.002115.

Teschner, J., N. Lachmann, J. Schulze, M. Geisler, K. Selbach, J. Santamaria-Araujo, J. Balk, R. R. Mendel, and F. Bittner. 2010. "A novel role for Arabidopsis mitochondrial ABC transporter ATM3 in molybdenum cofactor biosynthesis." Plant Cell 22 (2):468-80. doi: 10.1105/tpc.109.068478.

Tian, W., C. Chen, X. Lei, J. Zhao, and J. Liang. 2018. "CASTp 3.0: computed atlas of surface topography of proteins." Nucleic Acids Research 46 (W1):W363-W367. doi: 10.1093/nar/gky473. 
Wang, J., F. Sun, D. W. Zhang, Y. Ma, F. Xu, J. D. Belani, J. C. Cohen, H. H. Hobbs, and X. S. Xie. 2006. "Sterol transfer by ABCG5 and ABCG8: in vitro assay and reconstitution." Journal of Biological Chemistry 281 (38):27894-904. doi: 10.1074/jbc.M605603200.

Winn, M. D., C. C. Ballard, K. D. Cowtan, E. J. Dodson, P. Emsley, P. R. Evans, R. M. Keegan, E. B. Krissinel, A. G. Leslie, A. McCoy, S. J. McNicholas, G. N. Murshudov, N. S. Pannu, E. A. Potterton, H. R. Powell, R. J. Read, A. Vagin, and K. S. Wilson. 2011. "Overview of the CCP4 suite and current developments." Acta Crystallographica Section D Biological Crystallography 67 (Pt 4):235-42. doi: 10.1107/S0907444910045749.

Woo, J. S., A. Zeltina, B. A. Goetz, and K. P. Locher. 2012. "X-ray structure of the Yersinia pestis heme transporter HmuUV." Nature Structural \& Molecular Biology 19 (12):1310-5. doi: $10.1038 / \mathrm{nsmb} .2417$.

Zehnpfennig, B., I. L. Urbatsch, and H. J. Galla. 2009. "Functional reconstitution of human ABCC3 into proteoliposomes reveals a transport mechanism with positive cooperativity." Biochemistry 48 (20):4423-30. doi: 10.1021/bi9001908.

Zuo, J., Z. Wu, Y. Li, Z. Shen, X. Feng, M. Zhang, and H. Ye. 2017. "Mitochondrial ABC Transporter ATM3 Is Essential for Cytosolic Iron-Sulfur Cluster Assembly." Plant Physiology 173 (4):2096-2109. doi: 10.1104/pp.16.01760. 
bioRxiv preprint first posted online Nov. 9, 2019; doi: http://dx.doi.org/10.1101/836676. The copyright holder for this preprint (which was not peer-reviewed) is the author/funder, who has granted bioRxiv a license to display the preprint in perpetuity.

It is made available under a CC-BY 4.0 International license.

a
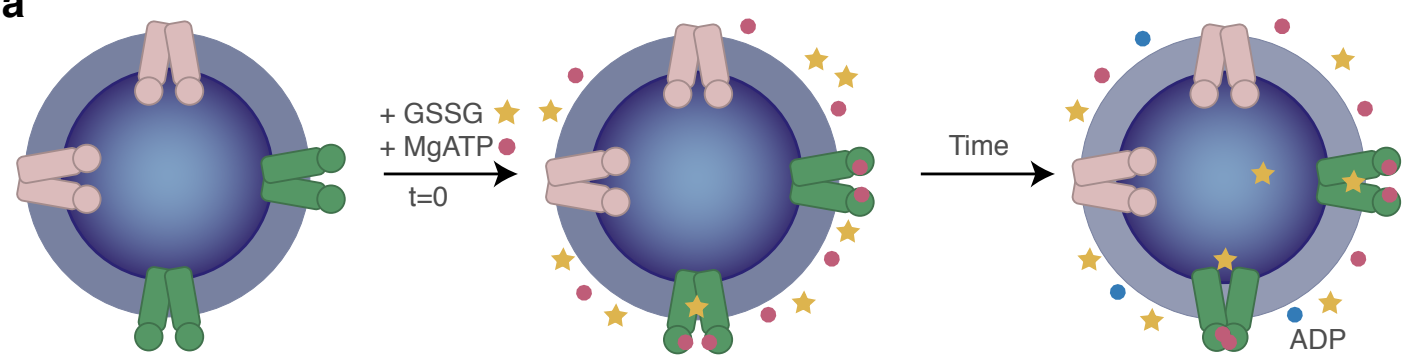

b
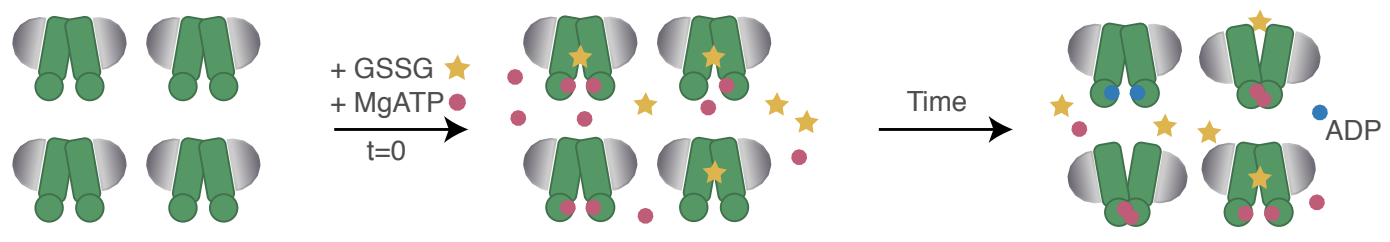

Figure 1. Functional assay schematics. (a) Transport and ATPase assay in proteoliposomes illustrating the two possible orientations of a transporter reconstituted in proteoliposomes. (b) ATPase assay in detergent. 
bioRxiv preprint first posted online Nov. 9, 2019; doi: http://dx.doi.org/10.1101/836676. The copyright holder for this preprint (which was not peer-reviewed) is the author/funder, who has granted bioRxiv a license to display the preprint in perpetuity.

It is made available under a CC-BY 4.0 International license.

a

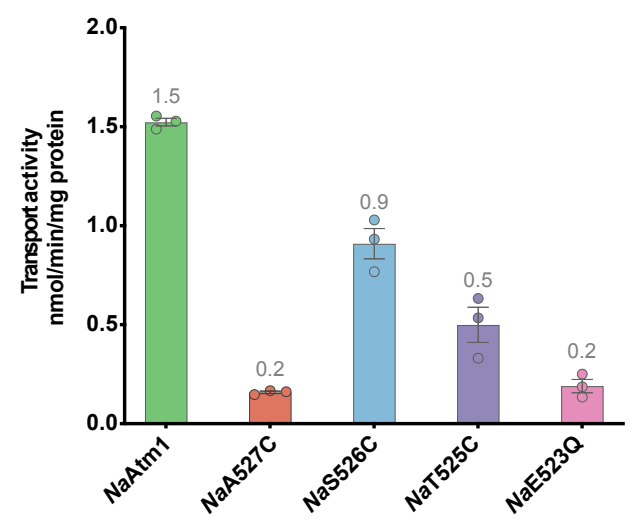

b

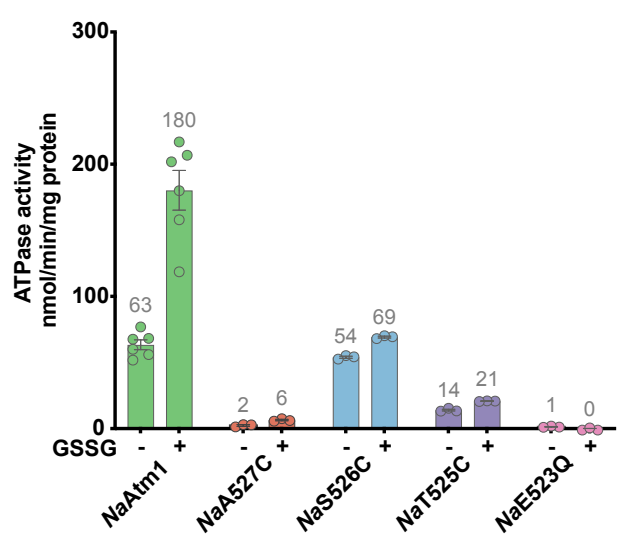

C

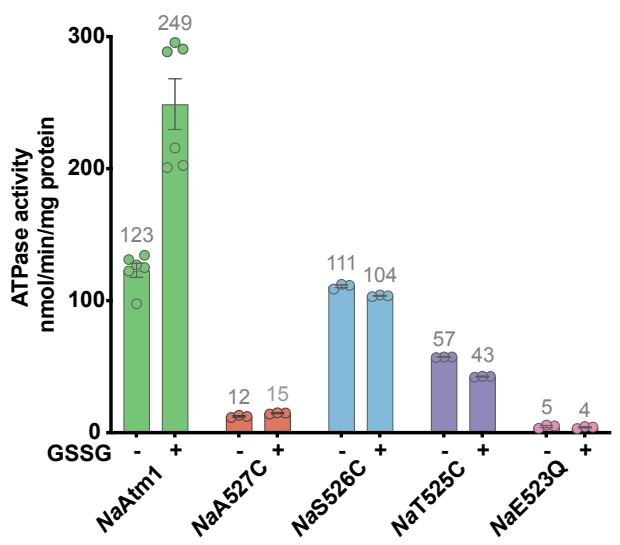

Figure 2. NaAtm1 transport and ATPase activities. (a) Transport activity of NaAtm1 and its variants with 10 mM MgATP and $2.5 \mathrm{mM}$ GSSG. (b, c) ATPase activities of NaAtm1 and its variants at $10 \mathrm{mM}$ MgATP in the absence and presence of $2.5 \mathrm{mM}$ GSSG, in PLS (b) and detergent (c). Error bars represent the standard error of the mean. Circles in $\mathbf{a}, \mathbf{b}$ and $\mathbf{c}$ represents the resuls of individual measurements. 
bioRxiv preprint first posted online Nov. 9, 2019; doi: http://dx.doi.org/10.1101/836676. The copyright holder for this preprint (which was not peer-reviewed) is the author/funder, who has granted bioRxiv a license to display the preprint in perpetuity.

It is made available under a CC-BY 4.0 International license.

a

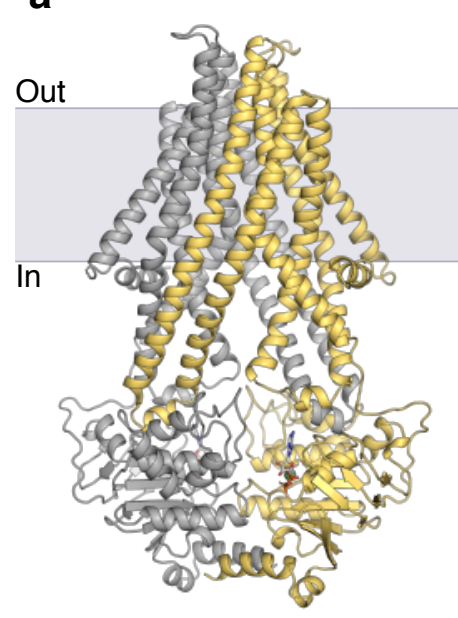

NaA527C1

inward occluded

MgADP b

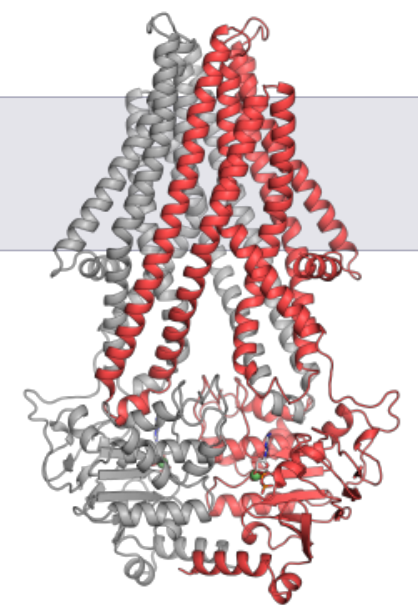

NaA527C2

inward occluded MgADP
C

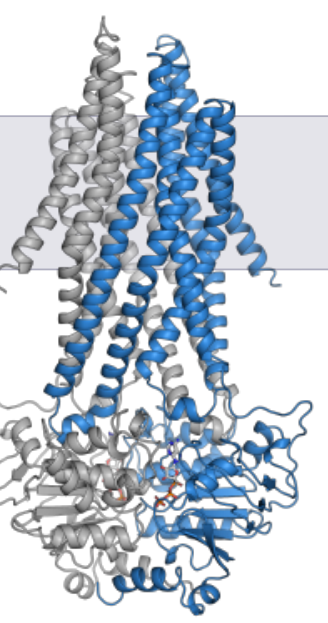

NaS526C

outward occluded

ATP d

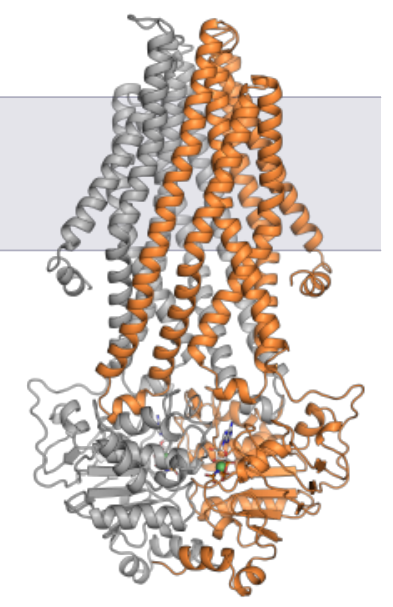

NaAtm1

occluded MgAMPPNP

Figure 3. Crystal structures of NaAtm1. (a,b) Structures of NaA527C in the inward-facing occluded conformation \#1 (a), and \#2 (b), both with MgADP bound. (c) Structure of NaS526C in the outward-facing occluded conformation with ATP bound. (d) Structure of wildtype NaAtm1 in a fully occluded conformation with MgAMPPNP bound. Each of the structures is colored with one chain in gray and the second chain in a different color. The corresponding nucleotides are shown in sticks with $\mathrm{Mg}^{2+}$ as green spheres. 
a

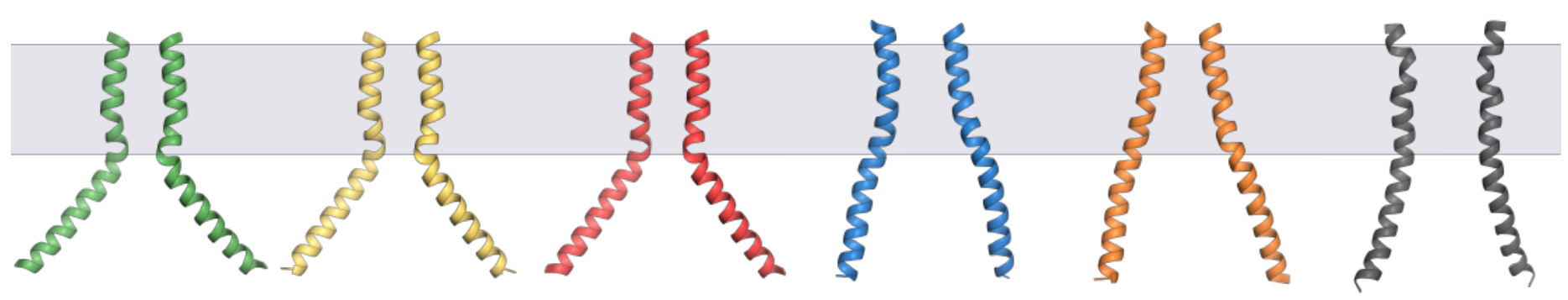

b

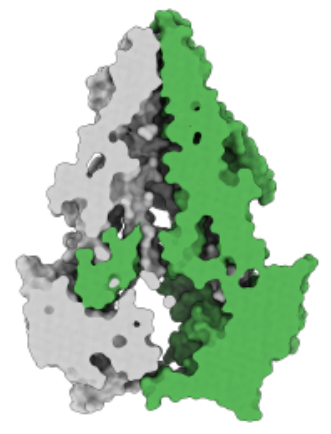

NaAtm1 inward-facing apo

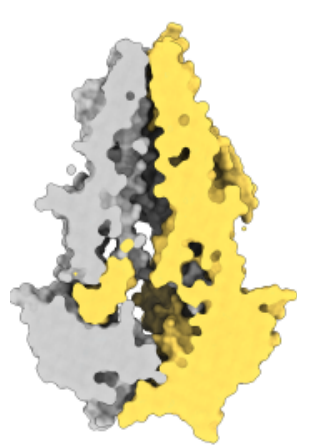

NaA527C1 inward occluded MgADP

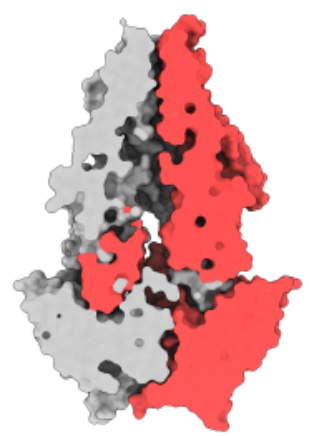

NaA527C2 inward occluded MgADP

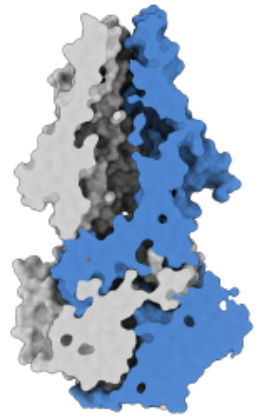

NaS526C outward occluded ATP
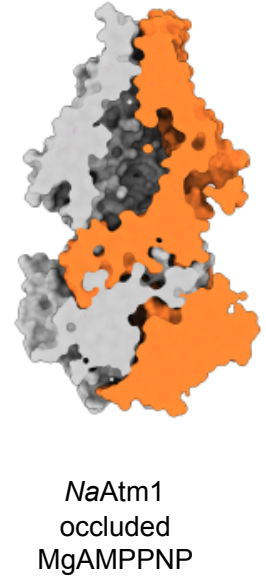
occluded
MgAMPPNP

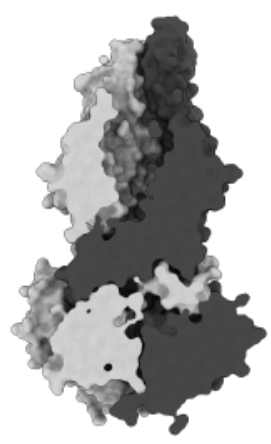

Sav1866 outward-facing ADP

Figure 4. Structural comparisons of NaAtm1 structures. (a) TM6 (residues 300-340) arrangements of all NaAtm1 structures in comparison to Sav1866 (residues 280-320). (b) Surface representations of the NaAtm1 structures in comparision to our earlier inward-facing structure (PDB ID: 4MRN) and the structure of Sav1866 in the outward-facing conformation (PDB ID: 2HYD) with a slice through the middle of the protein showing the sizes of the central cavities and possible substrate exit tunnels. NaAtm1 inward-facing structure (PDB ID: 4MRN) in green, NaA527C inward-occluded structure \#1 in yellow, NaA527C inward-occluded structure \#2 in red, NaS526C outward-occluded structure in blue, NaAtm1 occluded structure in orange and Sav1866 outward-facing structure (PDB ID: 2HYD) in black. 
bioRxiv preprint first posted online Nov. 9, 2019; doi: http://dx.doi.org/10.1101/836676. The copyright holder for this preprint (which was not peer-reviewed) is the author/funder, who has granted bioRxiv a license to display the preprint in perpetuity.

It is made available under a CC-BY 4.0 International license.

\begin{tabular}{|c|c|c|c|l|}
\hline Transporters & $\begin{array}{c}\text { ATPase activity } \\
\text { (nmol/min/mg) }\end{array}$ & $\begin{array}{c}\text { Transport Activity } \\
\text { (nmol/min/mg) }\end{array}$ & $\begin{array}{c}\text { Coupling } \\
\text { efficiency }\end{array}$ & \multicolumn{1}{|c|}{ References } \\
\hline ABCC3 & 200 & 1,200 & 0.17 & Zehnpfennig et al. 2009 \\
\hline MalFGK & $\sim 3$ & $\sim 2$ & $1.4-17$ & Davidson et al. 1990 \\
\hline OpuA & $\sim 80-120$ & $\sim 30-70$ & $1.7-4$ & Patzlaff et al. 2003 \\
\hline GInPQ & $15\left(\mathbf{m i n}^{-1}\right)$ & $8.5\left(\mathbf{m i n}^{-1}\right)$ & 1.8 & Lycklama et al. 2018 \\
\hline Pgp & $\sim 750-1,300$ & $\sim 500$ & 2 & Eytan et al. 1996 \\
\hline ABCG5/8 & 110 & 50 & 2.2 & Wang et al. 2006 \\
\hline MalFGK & $\sim 1.2-8$ & $\sim 0.3-2$ & $4-10$ & Dean et al. 1989 \\
\hline Pgp & $\sim 110$ & $\sim 6$ & 18 & Dong et al. 1996 \\
\hline HisP & 580 & 19 & 31 & Nikaido and Ames 1999 \\
\hline ABCG2 & $\sim 750$ & $\sim 22$ & 34 & Manolaridis et al. 2018 \\
\hline TmrAB & $\sim 1,100$ & $\sim 30$ & 37 & Hofmann et al. 2019 \\
\hline BtuCDF & $\sim 400$ & $\sim 4$ & 100 & Borths et al. 2005 \\
\hline HmuUV & $\sim 130$ & $\sim 1.1$ & 120 & Woo et al. 2012 \\
\hline NaAtm1 & 180 & 1.5 & 120 & This study \\
\hline MalFGK & 4,000 & 1.2 & 3,300 & Chen et al. 2001 \\
\hline ABCB6 & 610 & 0.03 & 20,000 & Chavan et al. 2013 \\
\hline
\end{tabular}

Table 1. Coupling efficiencies between ATP hydrolysis and substrate translocation for ABC transporters. Coupling efficiencies are either presented in the corresponding reference or calculated based on the reported ATPase and transport activities of the transporter. Coupling efficiency = ATPase activity/transport activity. 
bioRxiv preprint first posted online Nov. 9, 2019; doi: http://dx.doi.org/10.1101/836676. The copyright holder for this preprint (which was not peer-reviewed) is the author/funder, who has granted bioRxiv a license to display the preprint in perpetuity.

It is made available under a CC-BY 4.0 International license.

a

\begin{tabular}{|r|c|c|c|c|}
\hline & NaAtm1 & NaA527C & NaS526C & NaT525C \\
\hline & & \multicolumn{3}{|c|}{ ATPase activities $(\mathrm{nmol} / \mathrm{min} / \mathrm{mg})$} \\
\hline - GSSG (basal) & $64 \pm 4$ & $2.4 \pm 0.6$ & $54.0 \pm 0.8$ & $14.0 \pm 0.7$ \\
\hline + GSSG (total) & $180 \pm 15$ & $6.5 \pm 0.6$ & $69.3 \pm 0.7$ & $20.8 \pm 0.1$ \\
\hline Stimulated & $117 \pm 16$ & $4.1 \pm 0.8$ & $15 \pm 1$ & $6.8 \pm 0.7$ \\
\hline Transport rate & $1.52 \pm 0.02$ & $0.16 \pm 0.01$ & $0.91 \pm 0.08$ & $0.50 \pm 0.09$ \\
\hline & & Transport activities $(\mathrm{nmol} / \mathrm{min} / \mathrm{mg})$ \\
\hline Total/transport & $118 \pm 10$ & $41 \pm 4$ & $76 \pm 6$ & $42 \pm 7$ \\
\hline Stimulated/transport & $77 \pm 7$ & $26 \pm 6$ & $17 \pm 2$ & $14 \pm 3$ \\
\hline
\end{tabular}

b

\begin{tabular}{|r|c|c|c|c|}
\hline & NaAtm1 & NaA527C & NaS526C & NaT525C \\
\hline & & \multicolumn{3}{|c|}{ ATPase activities $\left(\mathrm{min}^{-1}\right)$} \\
\hline - GSSG (basal) & $8.4 \pm 0.5$ & $0.32 \pm 0.07$ & $7.2 \pm 0.1$ & $1.87 \pm 0.09$ \\
\hline + GSSG (total) & $24 \pm 2$ & $0.86 \pm 0.07$ & $9.21 \pm 0.09$ & $2.77 \pm 0.02$ \\
\hline Stimulated & $15.5 \pm 0.3$ & $0.5 \pm 0.1$ & $2.1 \pm 0.1$ & $0.90 \pm 0.09$ \\
\hline & & Transport activities $\left(\mathrm{min}^{-1}\right)$ & \\
\hline Transport rate & $0.203 \pm 0.003$ & $0.021 \pm 0.001$ & $0.12 \pm 0.01$ & $0.07 \pm 0.01$ \\
\hline
\end{tabular}

Table 2. ATPase and transport activities for wildtype NaAtm1 and variants. (a) ATPase and transport activities with calculated coupling efficiencies of wildtype NaAtm1 and variants in units of nmol/min $/ \mathrm{mg} .10$ mM MgATP and $2.5 \mathrm{mM}$ GSSG were used in for these measurements. (b) The same ATPase and transport activities as tabulated in (a) in units of $\mathrm{min}^{-1}$. All activities were measured three times except for wildtype ATPase activity, which was measured six times. 